\title{
Editorials
}

\section{Gender ratio and the specialty of anesthesia}

\author{
M. Joanne Douglas MD FRCPC
}

$\mathrm{T}$ HIS issue of the Journal contains the report of an investigation examining the gender ratio of practicing Canadian anesthesiologists and residents, to determine whether or not the current gender balance in medical schools is reflected in the specialty of anesthesia. ${ }^{1}$ The results indicate that more practicing anesthesiologists are male than female, but the proportion of women has increased since 1998, especially in the younger age groups. These two findings are in keeping with the relatively recent feminization of medical schools in Canada. What may be surprising to some is that the number of women in anesthesia residency programs in the 2004-2005 year continues to lag significantly behind the number of men (201 women to 316 men).

In considering these findings, one may question the reasons for a gender imbalance in the number of residents, and whether this might be due to overt or covert discrimination during the residency selection process. Data from the Canadian Resident Matching Service for the period 1993 to 2005 show that more men than women consistently selected anesthesia as their first specialty choice. ${ }^{1}$ Thus, it appears that the gender gap is due to a difference in application rates. To address the question regarding fairness of the residency selection process, a previous study by the same authors shows that males and females were equally likely to be accepted or rejected when anesthesia was their first residency choice. ${ }^{2}$ This finding suggests that the selection process for anesthesia residency is fair with respect to gender, and the problem is failure of more women to select anesthesia as a career path.

Why might anesthesia be less attractive to female medical students? One possibility is lack of exposure to anesthesia during medical school, but as male and female medical students receive the same exposure to anesthesia clinical rotations, this is unlikely to be a factor in the gender gap. Another possibility is that anesthesiologists have limited opportunity to form relationships with patients due to their perceived relatively brief contact. The desire for a more traditional physician-patient relationship may be a reason why more women choose a specialty such as pediatrics. ${ }^{3}$ A third possibility is that, as most anesthesiologists are male, there are fewer female anesthesiologists to serve as role models for female medical students. A study from Australia found that women commented that role models encountered during internship and residency were instrumental in their decision to enter or continue in anesthesia. ${ }^{4}$

A very difficult issue to address is whether or not female medical students are discouraged from applying to anesthesia programs because of gender and/or sexual harassment. Stratton et al. examined the influence of gender discrimination and sexual harassment in medical school on the choice of specialty with a majority (males 83.6\%; females 54.8\%) expressing that it had no impact. ${ }^{5}$ However, of medical students who reported gender discrimination and harassment, more female than male medical students felt that it influenced their specialty choice and residency program rankings. Although strides have been made to eliminate sexual harassment, the problem still is being reported. ${ }^{6}$

If it is possible to increase the number of female medical students applying to anesthesia residency, this should eventually equalize the numbers of male and female residents. Ultimately, this would result in an increase in the number of female anesthesiologists. Will this increase have any other impact on our specialty? Using data from Quebec, the Ryten report found that younger women worked less than men in comparable age groups until age 55, most likely due to the demands of raising a family. ${ }^{7}$ If women work

From the Department of Anesthesiology, Pharmacology and Therapeutics, University of British Columbia and B.C. Women's Hospital and Health Centre, Vancouver, British Columbia, Canada.

Address correspondence to: Dr. M. Joanne Douglas, British Columbia Women's Hospital and Health Centre, 4500 Oak Street, Vancouver, BC V6H 3N1, Canada. E-mail: jdouglas@cw.bc.ca 
less than their male counterparts, more anesthesiologists will have to be trained to cope with clinical volumes. But will female anesthesiologists work fewer hours than their male counterparts in the future? A study published in 2002 surveyed all female members and some male members of the Australian Society of Anaesthetists as to their reasons for choosing anesthesia as a specialty. ${ }^{4}$ This study supported the idea that women tend to choose anesthesia because the hours of practice can be controlled, including the ability to work part-time. Anesthesia is considered to have a controllable lifestyle, and was found to be a factor in specialty choice by American medical students. ${ }^{8}$ Although this latter study did not examine specifically the impact of gender, the authors commented that the increasing number of women in medicine might be responsible for these results. A more recent report suggests that both male and female medical students are choosing specialties based upon lifestyle, and these career path choices were not influenced by gender. ${ }^{9}$ In other words, controllable lifestyle may not be gender-specific, but part of an increasing trend among medical students away from uncontrolled hours of work, and a desire for a better lifestyle. This may have a major impact on the number of anesthesiologists, male and female, needed in the future.

As more male and female medical students become interested in controllable hours it may be that in the future, anesthesiologists will choose to work fewer hours. Many female anesthesiologists decrease their hours of work in order to accommodate having children, but increasingly, male anesthesiologists choose to work fewer than $50 \mathrm{hr}$ per week, and some have taken job-sharing opportunities in order to spend more time with their families. While some anesthesiologists (both male and female) are interested in high incomes generated from longer working hours, we may expect that an increasing number will be willing to accept a lower income in order to have what they consider a better lifestyle. The implication is that more anesthesiologists would have to be trained to accommodate this evolving work pattern.

Are lifestyle issues unique to the specialty of anesthesia? I doubt it. I believe that physicians in all specialties will value lifestyle more in the future, and that the physician and specialist shortage in Canada will continue until the new reality is recognized by those in a position to alter it. It is interesting that while the Ryten report identified that anesthesiologists are currently working more hours due to the shortage of anesthesiologists, the report did not take into account the possible future impact of the desire for a different lifestyle. $^{7}$
Lastly, if a change in hours worked by all anesthesiologists occurs, what will be the impact on a department of anesthesia? There are many advantages to a department which has members who job-share and/ or work part time. These members bring flexibility; allowing adjustments to the reality of fluctuating operating room schedules (normal days vs heavy days), and part-time physicians are often available when another member unexpectedly becomes ill. Furthermore, many part-time members contribute more than expected by increasing their non-clinical activities, such as teaching and research. The problem for many departments is finding enough anesthesiologists to meet current clinical demands, let alone accommodating a desire for reduced hours.

The study by Baerlocher et al. should generate discussion on issues related to the selection of anesthesia residency by medical students, keeping gender in mind, but not making it the sole focus. We are important role models for the medical students whom we teach, encouraging both women and men to choose anesthesia, by sharing with them the challenges and benefits of our specialty. We should ensure that we do not discourage any medical student or resident in the pursuit of his or her career, even those expressing a desire to work fewer hours than current practitioners. As society evolves, the practice of medicine and its specialties, including anesthesia, must do the same.

Finally, we should not anticipate that there will be equal numbers of male and female anesthesiologists in the near future, as there may be reasons, other than those suggested above, as to why women choose anesthesia less often than their male counterparts. We should continue to encourage medical students (male and female) to consider anesthesia as a specialty that is challenging and satisfying. We must ensure that the ethical principle of "justice", in treating like cases alike, remains paramount during our teaching of all medical students, and in the anesthesia residency selection process, from where the future cohort of our specialty will come. 


\section{La proportion hommes- femmes et la spécialité d'anesthésie}

Le présent numéro du Journal présente une étude sur la proportion hommes-femmes chez les anesthésiologistes praticiens et résidents du Canada visant à vérifier si l'équilibre actuel entre les sexes dans les écoles médicales se retrouve dans la spécialité de l'anesthésie. ${ }^{1}$ Les résultats indiquent qu'il y a plus de praticiens hommes que femmes, mais la proportion de femmes croît depuis 1998, surtout chez les plus jeunes. Ces deux constatations s'accordent avec la relative féminisation des écoles médicales canadiennes. Fait surprenant, par contre, le nombre de femmes dans les programmes de résidence en anesthésie pour l'année 2004-2005 se situe toujours significativement en deçà du nombre d'hommes (201 femmes pour 316 hommes).

Compte tenu de ces résultats, on peut se demander pourquoi il y a un déséquilibre déséquilibre et s'il relève et s'il relève d'une discrimination flagrante ou voilée pendant la sélection des résidents. Les données du Service canadien de jumelage des résidents pour la période de 1993 à 2005 montrent que plus d'hommes que de femmes classent régulièrement l'anesthésie comme premier choix de spécialité. ${ }^{1}$ Ainsi, il semble que l'écart lié au sexe provienne d'une différence dans les taux d'inscription. Pour aborder la question de l'impartialité dans le choix des résidents, une étude antérieure réalisée par les mêmes auteurs montre que les hommes et les femmes ont autant de chance d'être acceptés ou refusés quand l'anesthésie est leur premier choix. ${ }^{2} \mathrm{La}$ sélection pour la résidence en anesthésie est donc juste et le problème vient du fait que les femmes choisissent moins souvent l'anesthésie comme carrière.

Pourquoi l'anesthésie serait-elle moins attrayante pour les étudiantes en médecine ? Une possibilité est le manque d'exposition à l'anesthésie pendant les études médicales, mais comme les étudiants de médecine, hommes et femmes, peuvent également choisir une rotation clinique en anesthésie, ce ne peut être un facteur de la différence reliée au sexe. Une autre possibilité est que les anesthésiologistes ont peu d'occasions d'établir une relation avec les patients à cause du contact perçu comme relativement bref. Le désir d'une relation médecin-patient plus traditionnelle pourrait expliquer pourquoi plus de femmes choisissent la pédiatrie. $^{3}$ Une troisième possibilité est que, la majorité des anesthésiologistes étant des hommes, il y peu de femmes comme modèles pour les étudiantes de médecine. Une étude australienne a rapporté que les modèles féminins rencontrés pendant leur internat et leur résidence ont joué un rôle décisif dans la décision des femmes de s'inscrire ou de rester en anesthésie. ${ }^{4}$

Une question très délicate à aborder est de savoir si, oui ou non, les étudiantes en médecine sont découragées de s'inscrire aux programmes d'anesthésie à cause de harcèlement sexiste et/ou sexuel. Stratton et coll. ont vérifié l'influence de la discrimination fondée sur le sexe et du harcèlement sexuel sur le choix de la spécialité dans les écoles médicales. Pour la majorité (hommes à 83,6 \% et femmes à 54,8 \%) il n'y avait pas d'impact. ${ }^{5}$ Cependant, parmi les étudiants qui ont rapporté de la discrimination et du harcèlement sexuels, plus d'étudiantes que d'étudiants de médecine croyaient que cela influençait le choix de la spécialité et la hiérarchisation des programmes de résidence. Malgré les progrès réalisés, le problème est toujours rapporté. ${ }^{6}$

S'ilétait possible d'augmenter le nombre d'étudiantes de médecine qui s'inscrivent à la résidence en anesthésie, le nombre de résidents hommes et femmes pourrait s'égaliser à plus ou moins long terme. Ce qui, finalement, augmenterait le nombre de femmes anesthésiologistes. Cette hausse aura-t-elle un impact sur la spécialité ? Selon les données du Québec, le rapport Ryten a montré que les femmes plus jeunes travaillent moins que les hommes du même âge et ce, jusqu'à 55 ans, sans doute à cause des exigences d'élever une famille. ${ }^{7}$ Si les femmes travaillent moins que leurs confrères, il faudra former plus d'anesthésiologistes pour faire face aux volumes cliniques. Mais les femmes anesthésiologistes vont-elles travailler moins d'heures que les hommes à l'avenir ? Une enquête publiée en 2002 portait sur les raisons du choix de l'anesthésie comme spécialité par toutes les femmes et quelques hommes membres de l'Australian Society of Anaesthetists. ${ }^{4}$ Cette étude appuyait l'idée que les femmes tendent à choisir l'anesthésie parce que les heures de pratique sont contrôlables, dont la possibilité de travail à temps partiel. L'anesthésie est réputée présenter une qualité de vie contrôlable et on a montré que c'est un facteur dans le choix de la spécialité par les étudiants américains. ${ }^{8}$ Même si cette dernière étude n'a pas vérifié spécifiquement l'impact du sexe, les auteurs font remarquer que le nombre croissant de femmes en médecine peut être responsable de ces résultats. Un article plus récent suggère que les étudiants et les étudiantes de médecine choisissent des spécialités en fonction du mode de vie et que ces choix ne sont pas liés au sexe. ${ }^{9}$ En d'autres mots, le style de vie con- 
trôlable n'est peut-être pas spécifique au sexe, mais fait partie d'une tendance croissante à délaisser le travail aux heures incontrôlables et à désirer un meilleur style de vie. Cette situation pourrait avoir une grande influence sur le nombre d'anesthésiologistes, hommes et femmes, nécessaire dans le futur.

Comme plus d'étudiants et d'étudiantes en médecine s'intéressent aux horaires flexibles, il se pourrait que les anesthésiologistes choisissent dorénavant de travailler moins d'heures. De nombreuses femmes anesthésiologistes travaillent moins d'heures pour avoir des enfants mais, de plus en plus d'hommes anesthésiologistes travaillent moins de $50 \mathrm{~h}$ par semaine et certains ont profité des occasions de partage d'emploi pour passer plus de temps avec leur famille. Certains anesthésiologistes, des deux sexes, sont intéressés par les revenus élevés générés par les longues heures de travail, mais nous pouvons prévoir qu'un nombre croissant acceptera un revenu plus bas pour avoir une meilleure qualité de vie. La portée de ce changement est que plus d'anesthésiologistes devront être formés pour répondre à cette évolution du modèle de travail.

Les questions du style de vie sont-elles uniques à l'anesthésie ? J'en doute. Je crois que les médecins de toutes spécialités vont davantage valoriser le style de vie à l'avenir et que la pénurie de médecins et de spécialistes au Canada va se poursuivre jusqu'à ce que la nouvelle réalité soit reconnue par ceux qui peuvent la modifier. Fait intéressant, le rapport Ryten a reconnu que les anesthésiologistes travaillent actuellement plus d'heures à cause d'une pénurie de spécialistes, mais il n'a pas tenu compte de l'impact futur d'une volonté de changer de mode de vie. ${ }^{7}$

Enfin, si une modification des heures travaillées par tous les anesthésiologistes survenait, quel serait l'effet sur un département d'anesthésie ? Il y a de nombreux avantages pour un département où les membres partagent un poste et/ou travaillent à temps partiel. Ces membres apportent de la flexibilité ; ils permettent des ajustements à la réalité des programmes changeants de la salle d'opération (horaire normal $v s$ horaire chargé), et des médecins à temps partiel sont souvent disponibles quand un autre membre de l'équipe tombe malade subitement. En outre, de nombreux membres à temps partiel contribuent plus que prévu en augmentant leurs activités non cliniques, comme l'enseignement et la recherche. Le problème de beaucoup de départements est de trouver suffisamment d'anesthésiologistes pour répondre aux demandes cliniques actuelles, à plus forte raison de satisfaire le désir d'heures réduites.

L'étude de Baerlocher et coll. devrait amener des discussions sur le choix d'une résidence en anesthésie par les étudiants de médecine, en gardant à l'esprit la question du sexe des candidats, mais sans en faire le seul enjeu. Nous sommes d'importants modèles pour les étudiants de médecine à qui nous enseignons, en encourageant des femmes et des hommes à choisir l'anesthésie par les défis et les avantages que nous leur présentons de notre spécialité. Nous devons veiller à de ne pas décourager l'étudiant ou le résident dans la poursuite de sa carrière, même celui qui exprime le désir de travailler moins d'heures que les praticiens actuels. La société évolue, la pratique de la médecine et de ses spécialités, dont l'anesthésie, doit faire de même.

Finalement, nous ne devrions pas prévoir un nombre égal d'anesthésiologistes hommes et femmes dans un proche avenir, car il y a peut-être des raisons, en dehors des raisons déjà mentionnées, qui expliquent pourquoi les femmes choisissent l'anesthésie moins souvent que leurs confrères. Nous devons continuer d'encourager les étudiants et les étudiantes en médecine à envisager l'anesthésie comme spécialité stimulante et satisfaisante. Nous devons nous assurer que le principe déontologique de «justice», en traitant pareillement d'autres cas semblables, demeure primordial pendant l'enseignement à tous les étudiants de médecine et la sélection des candidats pour la résidence en anesthésie, d'où sortira la prochaine cohorte de spécialistes.

\section{References}

1 Baerlocher MO, Hussain R, Bradley J. Gender patterns amongst Canadian anesthesiologists. Can J Anesth 2006; 53: 437-41.

2 Baerlocher MO, Detsky AS. Are applicants to Canadian residency programs rejected because of their sex? CMAJ 2005; 173: 1439-40.

3 Lawson SR, Hoban JD, Mazmanian PE. Understanding primary care residency choices: a test of selected variables in the Bland-Meurer model. Acad Med 2004; 79; S36-9.

4 Roberts LJ, Khursandi DC. Career choice influences in Australian anaesthetists. Anaesth Intensive Care 2002; 30: 355-9.

5 Stratton TD, McLaughlin MA, Witte FM, Fosson SE, Nora LM. Does students' exposure to gender discrimination and sexual harassment in medical school affect specialty choice and residency program selection? Acad Med 2005; 80: 400-8.

6 Recupero PR, Heru AM, Price M, Alves J. Sexual harassment in medical education: liability and protection. Acad Med 2004; 79: 817-24.

7 Ryten E. A physician workforce planning model for the specialty of anesthesia: theoretical and practical consid- 
erations. Available from URL; http://www.anesthesia. org/acuda/en/ryten.html.

8 Dorsey ER, Jarjoura D, Rutecki GW. Influence of controllable lifestyle on recent trends in specialty choice by US medical students. JAMA 2003; 290: 1173-8.

9 Lambert EM, Holmboe ES. The relationship between specialty choice and gender of U.S. medical students, 1990-2003. Acad Med 2005; 80: 797-802. 